\title{
Some children with autism have latent social skills that can be tested
}

\author{
This article was published in the following Dove Press journal: \\ Neuropsychiatric Disease and Treatment \\ 16 March 2017 \\ Number of times this article has been viewed
}

\section{Michal Hrdlicka' \\ Tomas Urbanek ${ }^{2}$ \\ Maria Vacova' \\ Stepanka Beranova' \\ Iva Dudoval \\ 'Department of Child Psychiatry, Charles University Second Faculty of Medicine and University Hospital Motol, Prague, ${ }^{2}$ Institute of Psychology, Academy of Sciences, Brno, Czech Republic}

Correspondence: Michal Hrdlicka Department of Child Psychiatry, Charles University Second Faculty of Medicine, University Hospital Motol, V Uvalu 84, 15006 Prague, Czech Republic Tel +420 224433400

Fax +420 224433420

Email michal.hrdlicka@lfmotol.cuni.cz
Background: The idea of latent social skills in autism emerged as a possible interpretation of the rapid (but temporary) improvement of autistic subjects in oxytocin studies. We tested a hypothesis that a normal response to Item No 59 "Secure Base" from the third version of the Autism Diagnostic Interview - Revised (ADIR-59) could indicate the presence of latent social skills in autism.

Methods: We examined 110 autistic children ( 88 boys and 22 girls) with a mean age of $6.0 \pm 2.5$ years (range: $2.2-14.8$ years) using the Autism Diagnostic Observation Schedule (ADOS) Generic. A diagnosis of mental retardation was established in 68 autistic children (62\%).

Results: The difference in the ADOS social domain between children aged $\leq 5$ years on one side and children older than 5 years on the other side was significant in subjects with normal responses to ADIR-59 (9.60 vs $6.47 ; P=0.031)$ but not in those with abnormal responses to ADIR-59 (10.62 vs 9.63; $P=0.537)$. In a predictive model, lower ADOS social domain scores were predicted by older age $(P=0.001)$, lower scores on the ADIR-59 $(P=0.01)$, and the absence of mental retardation $(P=0.049)$.

Conclusion: The results support the hypothesis that the normal response to item ADIR-59 "Secure Base" indicates the presence of latent social skills in autism that might foretell further social growth in older autistic subjects.

Keywords: autism, latent social skills, Autism Diagnostic Interview - Revised, Autism Diagnostic Observation Schedule, prediction

\section{Introduction}

Autism spectrum disorder (ASD), according to the Diagnostic and Statistical Manual of Mental Disorders, Fifth Edition (DSM-5), ${ }^{1}$ or ASDs, according to the International Classification of Diseases, Tenth Edition (ICD-10), ${ }^{2}$ have generally been regarded as life-long conditions. However, some questions about the nature of ASD were exposed by oxytocin studies, which have been carried out over the past decade.

Several studies with oxytocin in ASD adults demonstrated significant improvement in social cognition, ${ }^{3-7}$ although studies in ASD children and adolescents brought rather disappointing results. ${ }^{8,9}$ If the codified concept of describing ASDs as disorders with severe, pervasive abnormalities in reciprocal social interaction and life-long impairment is unequivocally true, then the interpretation of rapid (but temporary) improvement with oxytocin is difficult at best. ${ }^{10}$ Andari et $\mathrm{al}^{4}$ suggested that "patients with autism might possess latent social skills, and thus oxytocin might favor social engagement behavior by suppressing fear and mistrust". This explanation was both fair and acceptable but surprisingly has aroused negligible attention.

A central question of the concept that (at least some) people with autism may have latent social skills is how to reveal and test social skills that are hidden. In other 
fields of psychiatry, projective tests such as the Rorschach inkblot test proved useful in revealing hidden characteristics of the mind; however, they were not designed to uncover hidden abilities, and their contribution to the field of ASD has been generally limited. ${ }^{11}$ The Theory of Mind, ${ }^{12}$ a specific approach regarding how to test the autistic mind, relies predominantly on social abilities that the child is able and/ or willing to express.

The immanent feature of hypothetical latent social skills seems to be that they are not accessible by direct observation; otherwise, they would have already been discovered during more than seven decades of autism research. Thus, we are left with indirect observation as the only viable option. An indirect approach to observation of subtle phenomena has been widely used in physics and astronomy but rather less used in medicine, eg, undetectable particles are observed through their impact on trajectories of detectable particles or invisible planetoids are observed through their impact on trajectories of visible planets. There have been, although rare, examples of using such an approach in psychiatry, eg, Nancy Andreasen discovered the negative symptoms of schizophrenia while she was studying why schizophrenic patients who had formally been in remission according to former criteria were unable to work (Andreasen, personal communication, November 1997). The indirect approach, in our case, means searching for potential nonstandard (eg, stressful or threatening) situations where hypothetical latent social skills would impact the behavior of the child, and through this behavioral impact would become detectable.

For methodological reasons, we decided to explore for our goals an existing assessment instrument with known validity and reliability, instead of creating a new assessment tool. We performed a "text" analysis of the most precise standardized interview, the Autism Diagnostic Interview Revised (ADI-R), ${ }^{13}$ to determine whether any of its items were suitable for testing the latent social skills hypothesis. We identified in the third version of the ADI-R, which appeared in 1995, item number 59 "Secure Base" (Supplementary material). This item has been removed from the current version of ADI-R. ${ }^{14}$

ADI-R 59 “Secure Base," with assessment of 4-5 year olds or to the current status in younger children, is pertinent to separation anxiety, although indirectly. A developmentally appropriate (normal) response in typically developing children involves a child that can play apart from his/her caregivers; however, he/she is able to foresee that permanent separation from parents or caregivers would have a catastrophic impact on his/her life situation. Thus, he/she regularly "checks back" to verify the proximity of parents or caregivers. In autistic children, various degrees of abnormal response are offered (Supplementary material). We suggest that a normal response to the "Secure Base" item could indicate the presence of latent social skills in autism.

The aim of our study was to test the hypothesis that ADI-R No 59 "Secure Base" could point to latent social skills in autism. For this purpose, we established two criteria for how to test whether the item is truly related to latent social skills: 1) performance on the item revealing latent social skills should correlate with expressed social skills according to the iceberg principle, ie, the extent of latent social skills should correspond with expressed social skills, and 2) performance on the item should have predictive value for further social development, ie, if the concept of latent social skills is valid, it should also represent the possibility of future social growth.

\section{Methods \\ Participants}

The sample consisted of 154 children (mean age at examination: $6.5 \pm 2.7$ years; 120 boys and 34 girls) who attended a diagnostic examination focused on autism at the Department of Child Psychiatry. Children were consecutively recruited from November 2012 to June 2016.

A diagnosis of ASD was confirmed in 110 children ( $71 \%$ of the sample; 88 boys and 22 girls), and it was only the "confirmed" group's data that were used in most of the analyses presented in the Results section. The mean age at examination of children with a confirmed ASD diagnosis was 6.0 2 2.5 years (range: $2.2-14.8$ years). Diagnoses, based on the ICD-10, included 85 patients with childhood autism, 21 patients with atypical autism, 3 patients with Asperger syndrome, and 1 patient with other childhood disintegrative disorder. In 68 autistic patients (62\%), a diagnosis of mental retardation (MR) was also established. Of these, 32\% had mild MR, 40\% had moderate MR, 4\% had severe MR, and $24 \%$ had unspecified MR. In 33 autistic patients (30\%), a hyperkinetic syndrome was noted but did not involve a formal diagnosis (ICD-10 still does not allow a diagnosis of both ASD and hyperkinetic disorder together.).

The diagnoses in the 44 children, where the ASD diagnosis was ruled out, included specific developmental disorders of speech and language (15 cases), hyperkinetic disorder (12 cases), MR (12 cases), abnormal personality development (3 cases), hyperkinetic conduct disorder (3 cases), conduct disorder ( 2 cases), and other childhood emotional disorders ( 2 cases). Other mixed disorders of conduct and emotions, 
phobic anxiety disorder of childhood, combined vocal and multiple motor tic disorder, and adjustment disorder were represented by one case of each. Several patients suffered from combined diagnoses. This group served as a control group but only for the first analysis in the Results section.

\section{Procedure}

This study was approved by the multicenter ethics committee of the University Hospital Motol. Parents who agreed to having their child participate in the study signed informed consents.

The ICD- $10^{2}$ was used to make the clinical diagnoses. The assessment involved testing using the third version of the ADI-R, ${ }^{13}$ the Autism Diagnostic Observation Schedule (ADOS) - Generic, ${ }^{15}$ and a clinical examination by two experienced child psychiatrists with expertise in autism. All raters and examiners were blinded to the tested hypotheses. The concept of the best-estimate clinical diagnosis (BECD), by consensus of two experienced specialists, was used as the gold standard. ${ }^{16}$ If there was disagreement between the ADI-R or ADOS diagnosis and the BECD, the latter was preferred. IQ testing was also performed. Gesell Developmental Scale was used for the youngest children, and the Stanford-Binet Intelligence Scale, Fourth Edition, was used for older children.

For further data analysis, the social interaction total score of ADOS (in the following text labeled as ADOSsoc) was used. Based on the suggested diagnostic algorithm for ICD-10, the ADI-R social domain, communication domain, and restrictive interests and repetitive behavior domain were used.

ADI-R No 59 "Secure Base" (in the following text labeled as ADIR-59) was for some calculations dichotomized so that a normal response (code " 0 ") remained unchanged, and abnormal responses (codes " 1,2 , or 3") were collapsed into one value. This dichotomized variable was labeled ADIR-59R. It is worth noting that ADI-R No 59 was not included in any of the three domains (ie, social, communication, and restrictive interests and repetitive behavior domains) of the ICD-10-suggested diagnostic algorithm based on the ADI-R. Thus, ADI-R No 59 could be correlated, as an independent variable, with the main ADI-R domains, which can serve as baseline values since they measure symptoms at the same age as ADIR-59.

\section{Data analysis}

Statistical analysis was performed using the Statistical Package for the Social Sciences (IBM SPSS, version 22.0) and statistical software $\mathrm{R}^{17}$ with the psych package. ${ }^{18}$ Descriptive statistics for the samples were used. The chi-square test with Yates' continuity correction was used for comparison of the proportion of normal and abnormal responses on the dichotomized ADIR-59R variable between the autistic and the control groups. The Spearman correlation analysis was used to explore relationships between the ADIR-59 variable and ADI-R domains as well as, with adjustment, for multiple testing using the Holm-Bonferroni correction for exploratory analysis in a prediction model. Differences in the mean ADI-R social domain and the ADOS-soc regarding gender (boys vs girls) and age (between aged children $\leq 5$ years vs children $>5$ years) were studied using the independent samples Mann-Whitney $U$-test. Differences in the mean ADOS-soc between children aged $\leq 5$ years and children $>5$ years, separately for normal and abnormal responses to ADIR-59, were also analyzed using the independent samples Mann-Whitney $U$-test. The chi-square test was used to test for an agreement of the prediction model with the data. Path analysis was used in the prediction model.

\section{Results \\ Analysis of the ADI-R No 59 "Secure Base"}

The proportion of normal and abnormal responses on the dichotomized ADIR-59R variable did not differ significantly between the autistic group (32 vs 53) and the control group ( 18 vs $14 ; \chi^{2}=2.571, d f=1, P=0.109$ ). This result shows that an abnormal response on ADIR-59R was not necessarily specific for autism but could also be seen in the non-autistic clinical population, which served as controls in this comparison.

All following analyses were performed on the autistic group only. The ADIR-59 correlated significantly with the ADI-R social domain $(R=0.433, P<0.001)$ but not with the communication domain $(R=0.121, P=0.270)$ or the restrictive interests and repetitive behavior domain $(R=-0.042$, $P=0.705)$.

There were no differences between boys and girls in either the ADI-R social domain (23.3 vs 26.3; $\mathrm{n}=96, U=930.0$, $P=0.206)$ or the ADOS-soc (9.16 vs $9.53 ; \mathrm{n}=87, U=640.5$, $P=0.624)$; thus, we did not do any further analyses involving gender.

When we compared children aged $\leq 5$ years on one side and children aged $>5$ years on the other side, the mean ADI-R social domains did not differ between the two age groups ( 25.47 vs 22.72 ; $\mathrm{n}=96, U=884.5, P=0.06$ ), whereas the ADOS-soc was different ( 10.49 vs $8.21 ; \mathrm{n}=87, U=597.0$, $P=0.004)$. This was an expected result since the ADI-R scores 
represent historical baseline values in older children, while the ADOS scores reflect the child's current status.

The difference in the mean ADOS-soc between children aged $\leq 5$ years on one side and children aged $>5$ years on the other side was significant in children with normal responses on ADIR-59R (9.60 vs 6.47; $\mathrm{n}=27, U=42.0, P=0.031$ ) but not in children with abnormal responses on ADIR-59R (10.62 vs 9.63; $\mathrm{n}=40, U=176.0, P=0.537$ ). Figure 1 shows the results expressed alternatively as median values with $95 \%$ confidence intervals of the medians. This result favors the children with normal responses on ADIR-59R and suggests that these children are more likely to have better social skills by the time they reach school age.

\section{Predictive model}

We calculated a correlation matrix for all study variables used for hypothesis testing, and their intercorrelations with probability values are presented in Table 1 .

We built a path analysis model to test mediation between age and ADOS social domain using ADI-R No 59 with MR as a separate predictor of the ADOS social domain (Figure 2). The model fit was not statistically significant $\left(\chi^{2}=1.389, d f=1\right.$, $P=0.239$ ), which implies good reproduction of the correlation matrix entering the analysis. The results of the path analysis are presented in Table 2.

The ADOS-soc was predicted using the age of the patient $(P=0.001)$, response on ADIR-59 $(P=0.01)$, and MR $(P=0.049)$, ie, lower ADOS-soc scores were significantly more likely in older children, in lower scores (normal or mildly abnormal) on the ADIR-59, and in the absence of MR.

5 years or younger

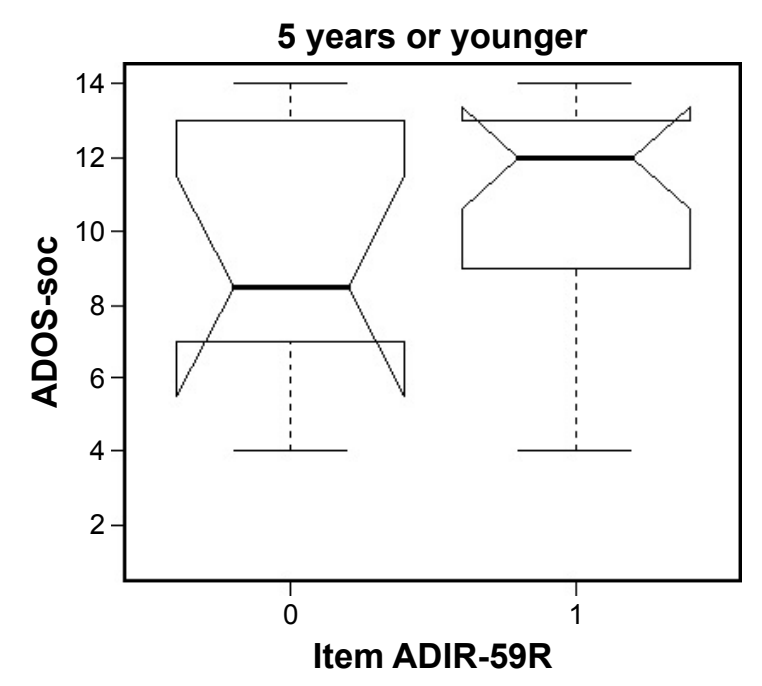

The proportion of variance in the ADOS-soc accounted for by all predictors was 0.158 . The indirect effect of age on the ADOS-soc through ADIR-59 was not significant $(P=0.568)$.

\section{Discussion}

In our opinion, the results support the hypothesis that item ADIR-59 "Secure Base" is related to latent social skills. Neither criterion, which was established to test the hypothesis, was rejected by statistical testing. The first criterion was that performance on the item revealing latent social skills should correlate with expressed social skills according to the iceberg principle. In fact, ADIR-59 item correlated significantly high with ADI-R social domain but not with other ADI-R domains. The second criterion was that the performance on the item should have predictive value for social development. In our predictive model, item ADIR-59 and age predicted better result in ADOS-soc and MR predicted a worse score.

Many prediction studies for ASD have been performed; however, to our knowledge, no prediction study included ADIR-59 "Secure Base" or other measure of developmentally appropriate separation anxiety. Some of the studies found that MR (or intellectual disability in terms of the DSM-5) predicted poorer outcomes, ${ }^{19-22}$ which agrees with our findings. Our observation that the ADOS social domain scores decrease with age corresponds with renowned reviews showing that by school age, many children with autism become more responsive socially, ${ }^{23}$ and reduction in ADOS social domain scores and repetitive domain scores were observed, although there were few changes in the communication domain scores. ${ }^{24}$

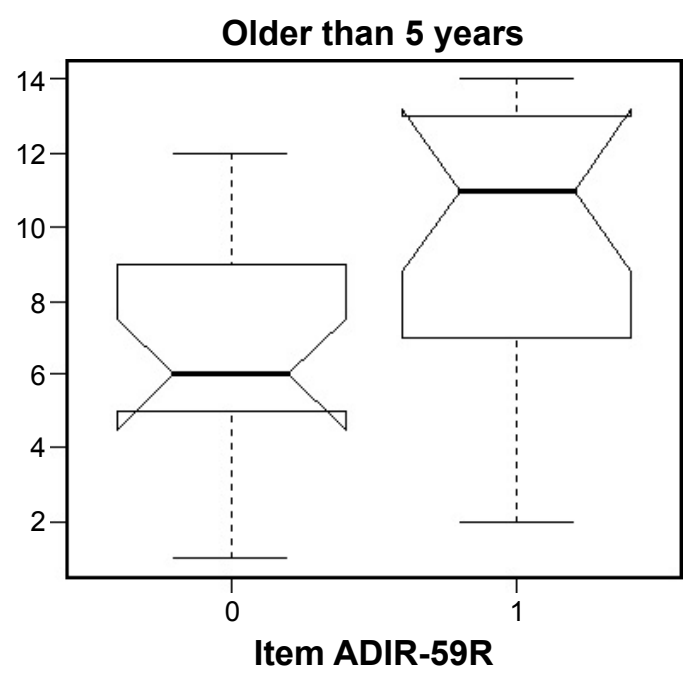

Figure I Differences in the median ADOS-soc between children aged $\leq 5$ years and children aged $>5$ years relative to normal and abnormal responses on ADIR-59R. Notes: Boxplots show median values and both lower and upper quartiles. The "notches" display $95 \%$ confidence intervals of the medians.

Abbreviations: ADIR-59R, dichotomized No 59 "Secure Base" (normal vs abnormal response) from the third version of the Autism Diagnostic Interview - Revised; ADOS-soc, social interaction total score of Autism Diagnostic Observation Schedule - Generic. 
Table I Correlation matrix of study measures

\begin{tabular}{llll}
\hline Variables & Age & ADOS-soc & ADIR-59 \\
\hline ADOS-soc & $-0.38^{* * *}$ & & \\
ADIR-59 & -0.09 & $0.32^{* *}$ & \\
MR & -0.10 & $0.27^{*}$ & 0.16 \\
\hline
\end{tabular}

Notes: Number of patients entering correlation analysis $=67 . * P<0.05, * * P<0.01$, $* * * P<0.001$. After adjustment for multiple testing using the Holm-Bonferroni correction, only correlation of ADOS-soc with age remained significant $(P<0.0 \mathrm{I})$. Abbreviations: ADIR-59, item No 59 "Secure Base" from the third version of the Autism Diagnostic Interview - Revised; ADOS-soc, social interaction total score of Autism Diagnostic Observation Schedule - Generic; MR, mental retardation.

A more conservative and an alternative explanation of our results would be that ADIR-59 measures separation anxiety only and is not related to latent social skills. Then, the interpretation of ADIR-59 would suggest that the presence of developmentally appropriate, nonpathological separation anxiety (ADIR-59 code "0") at 4-5 years of age and younger predicts better social development of children with ASD by the time they reach school age. Generally, anxiety in ASD has become a topical issue of research in the past decade. Prevalence rates of anxiety disorders in autistic children are much higher $(\sim 40 \%)$ than in typically developing children. ${ }^{25}$ However, it has also been observed that children with anxiety disorders have higher scores for both ASD traits in early development and current ASD symptoms than typically developing children. ${ }^{26}$ Among high-functioning ASD youth referred for anxiety, $41.7 \%$ had social phobia, $25.9 \%$ generalized anxiety disorder, $15.7 \%$ separation anxiety disorder, $12.0 \%$ obsessive-compulsive disorder, and $4.6 \%$ specific phobia. Overall, $91.6 \%$ met the criteria for two or more anxiety disorders. ${ }^{27}$ Structural equation modeling suggested that anxiety symptoms experienced by children with ASD are separate from ASD symptom severity and may instead reflect anxiety syndromes (eg, separation anxiety) similar to those that occur in typically developing children..$^{28}$

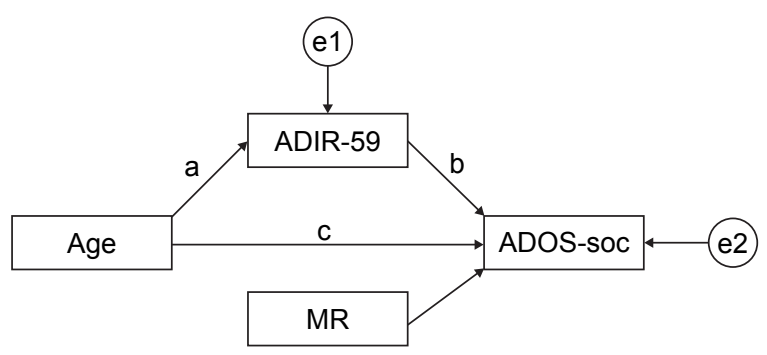

Figure 2 Path analysis model.

Notes: e1, e2, residual variables.

Abbreviations: ADIR-59, No 59 "Secure Base" from the third version of the Autism Diagnostic Interview - Revised; ADOS-soc, social interaction total score of Autism Diagnostic Observation Schedule - Generic; MR, mental retardation.
Table 2 Path analysis model for test of mediation between age and ADOS social domain through ADI-R No 59 and MR as a separate predictor

\begin{tabular}{lllll}
\hline Paths/coefficients & Estimate & $\begin{array}{l}\text { Standard } \\
\text { error }\end{array}$ & z-value & P-value \\
\hline Regressions & & & \\
$\quad$ ADOS-soc $\approx$ age $(c)$ & -0.585 & 0.175 & -3.343 & $\mathbf{0 . 0 0 1}$ \\
$\quad \sim$ MR & 1.574 & 0.800 & 1.967 & $\mathbf{0 . 0 4 9}$ \\
$\quad$ ADIR-59 $\approx$ age $(a)$ & 0.037 & 0.063 & 0.586 & 0.558 \\
$\quad$ ADOS-soc $\approx$ ADIR-59 (b) & 0.878 & 0.339 & 2.590 & $\mathbf{0 . 0 1 0}$ \\
Effects in the model & & & & \\
$\quad$ Indirect effect (ab) & 0.032 & 0.057 & 0.572 & 0.568 \\
$\quad$ Total (ab + c) & -0.553 & 0.183 & -3.020 & $\mathbf{0 . 0 0 3}$ \\
\hline
\end{tabular}

Notes: Significant results are in bold. Variances of the residual variables: el $=\mid .352$, $P<0.00 \mathrm{I} ; \mathrm{e} 2=10.398, P<0.00 \mathrm{I}$. el, e2, residual variables.

Abbreviations: ADI-R, Autism Diagnostic Interview - Revised; ADIR-59, Item No 59 "Secure Base" from the third version of the Autism Diagnostic Interview Revised; ADOS, Autism Diagnostic Observation Schedule; ADOS-soc, social interaction total score of Autism Diagnostic Observation Schedule - Generic; MR, mental retardation.

To summarize, this part of the "Discussion" section was devoted to alternative interpretations of our results; however, we still believe that ADIR-59 involves more than just an assessment of developmentally appropriate separation anxiety. A normal response on the item means that the autistic child has separation anxiety, but he/she is able to correct it using his/her own social skills. Therefore, the item correlated highly significantly with the ADI-R social domain as was presented in the Results section. If the item was only related to separation anxiety, we would not expect any significant correlation.

Some parallels can be seen between our results and the Theory of Mind-based research on deception and sabotage in autistic children. Sodian and Frith ${ }^{29}$ found that autistic children performed poorly on a deception task (ie, telling a lie) but performed well on a sabotage task (ie, obstruct an opponent). Their success on the sabotage task demonstrated that their failure at deception was not due to an inability to understand the task. The authors concluded that good performance on the simple sabotage task was evidence for an ability to control a behavioral tendency when no belief manipulation was involved. Although the term latent social skills was not used, better performance results on the behavioral social task compared to the verbal social task correspond partly with both our presumptions and results.

Our findings are limited by of a cross-sectional study that examined children at the age of their first autism diagnosis. Thus, the study could not assess the influence of any therapeutic interventions on social development. Another limitation of our study is that it focused on psychopathological and behavioral aspects only and did not involve genetic 
or neurobiological measures that are now major topics in autism research. ${ }^{30-33}$

Finally, our study had a relatively small sample size. Larger and differently designed studies are needed to validate our results, and it can be done in three ways:

1. longitudinal follow-up studies focused on developmental trajectories in autism that include ADIR-59 and the effect of therapeutic interventions in the predictive model;

2. studies on recovery from autism that take ADIR-59 into account; and

3. oxytocin studies that classify the response to oxytocin relative to ADIR-59.

Nonetheless, our observation of latent social skills through ADIR-59 was indirect, and ADIR-59, per se, seems to be more of an indicator than a measure of latent social skills. To better validate the concept of latent social skills, development of a new scale for measuring latent social skills would be a great goal for future research.

\section{Conclusion}

The results support the hypothesis that the normal response to item ADIR-59 "Secure Base" indicates the presence of latent social skills in autism that might foretell further social growth in older autistic subjects. In our opinion, the concept of latent social skills could represent a new dimension in autistic symptoms, a dimension that might be worthy of future elaboration and one that might prove essential for predicting future development of autistic children and for better focused therapeutic interventions.

\section{Acknowledgments}

This study was supported by grant no 16-31754A and project for conceptual development of research organization 00064203 (University Hospital Motol, Prague, Czech Republic) of the Ministry of Health of the Czech Republic. All rights reserved. The authors thank Thomas Secrest, MSc, for his assistance with the English version of the manuscript.

\section{Disclosure}

The authors report no conflicts of interest in this work.

\section{References}

1. American Psychiatric Association. Diagnostic and Statistical Manual of Mental Disorders (DSM-5). Arlington, VA: American Psychiatric Publishing; 2013.

2. World Health Organization. International Classification of Diseases. 10th ed. Geneva: WHO; 1992.

3. Hollander E, Bartz J, Chaplin W, et al. Oxytocin increases retention of social cognition in autism. Biol Psychiatry. 2007;61(4):498-503.
4. Andari E, Duhamel JR, Zalla T, Herbrecht E, Leboyer M, Sirigu A. Promoting social behavior with oxytocin in high-functioning autism spectrum disorders. Proc Natl Acad Sci U S A. 2010;107(9): 4389-4394.

5. Guastella A, Einfeld SL, Gray KM, et al. Intranasal oxytocin improves emotion recognition for youth with autism spectrum disorders. Biol Psychiatry. 2010;67(7):692-694.

6. Anagnostou E, Soorya L, Chaplin W, et al. Intranasal oxytocin versus placebo in the treatment of adults with autism spectrum disorders: a randomized controlled trial. Mol Autism. 2010;3(1):16.

7. Watanabe T, Kuroda M, Kuwabara H, et al. Clinical and neural effects of six-week administration of oxytocin on core symptoms of autism. Brain. 2015;138(pt 11):3400-3412.

8. Dadds MR, MacDonald E, Cauchi A, Williams K, Levy F, Brennan J. Nasal oxytocin for social deficits in childhood autism: a randomized controlled trial. J Autism Dev Disord. 2014;44(3):521-531.

9. Guastella AJ, Gray KM, Rinehart NJ, et al. The effects of a course of intranasal oxytocin on social behaviors in youth diagnosed with autism spectrum disorders: a randomized controlled trial. J Child Psychol Psychiatry. 2015;56(4):444-452.

10. Hrdlicka M, Dudova I. Controversies in autism: is a broader model of social disorders needed? Child Adolesc Psychiatry Ment Health. 2013;7(1):9.

11. Klin A, Saulnier C, Tsatsanis K, Volkmar FR. Clinical evaluation in autism spectrum disorders: psychological assessment within a transdisciplinary framework. In: Volkmar FR, Paul R, Klin A, Cohen D, editors. Handbook of Autism and Pervasive Developmental Disorders. 3rd ed. New York: John Wiley \& Sons; 2005:772-798.

12. Baron-Cohen S, Leslie AM, Frith U. Does the autistic child have a "theory of mind"? Cognition. 1985;21(1):37-46.

13. Lord C, Rutter M, LeCouteur A. Autism diagnostic interview - revised: a revised version of a diagnostic interview for caregivers of individuals with possible pervasive developmental disorders. J Autism Dev Disord. 1994;24(5):659-685.

14. Rutter M, LeCouteur A, Lord C. ADI-R: The Autism Diagnostic Interview-Revised. Los Angeles, CA: Western Psychological Services; 2003.

15. Lord C, Risi S, Lambrecht L, et al. The autism diagnostic observation schedule-generic: a standard measure of social and communication deficits associated with the spectrum of autism. J Autism Dev Disord. 2000;30(3):205-223.

16. Kim SH, Lord C. Combining information from multiple sources for the diagnosis of autism spectrum disorders for toddlers and young preschoolers from 12 to 47 months of age. J Child Psychol Psychiatry. 2012; 53(2): $143-151$.

17. $\mathrm{R}$ Core Team [homepage on the Internet]. $R$ : A Language and Environment for Statistical Computing. Vienna, Austria: R Foundation for Statistical Computing; 2015. Available from: http://www.R-project. org/. Accessed October 21, 2016.

18. Revelle W [webpage on the Internet]. Psych: Procedures for Personality and Psychological Research. Version $=1$ 1.5.6. Evanston, IL: Northwestern University; 2015. Available from: http://CRAN.R-project.org/ package=psych. Accessed October 21, 2016.

19. Baghdadli A, Assouline B, Sonie S, et al. Developmental trajectories of adaptive behaviors from early childhood to adolescence in a cohort of 152 children. J Autism Dev Disord. 2012;42(7): 1314-1325.

20. Fountain C, Winter AS, Bearman PS. Six developmental trajectories characterize children with autism. Pediatrics. 2012;129(5): e1112-e1120.

21. Smith LE, Maenner MJ, Seltzer MM. Developmental trajectories in adolescents and adults with autism: the case of daily living skills. J Am Acad Child Adolesc Psychiatry. 2012;51(6):622-631.

22. Hedvall A, Westerlund J, Fernell E, et al. Preschoolers with autism spectrum disorder followed for 2 years: those who gained and those who lost the most in terms of adaptive functioning outcome. J Autism Dev Disord. 2015;45(11):3624-3633. 
23. Volkmar FR, Lord C, Klin A, Schultz R, Cook EH. Autism and the pervasive developmental disorders. In: Martin A, Volkmar FR, editors. Lewis's Child and Adolescent Psychiatry: A Comprehensive Textbook 4th ed. Philadelphia: Lippincott Williams \& Wilkins; 2007:384-400.

24. Charman T. Development from preschool through school age. In Amaral DG, Dawson G, Geschwind DH, editors. Autism Spectrum Disorders. New York: Oxford University Press; 2011:229-240.

25. Steensel FJA, van Bogels SM, Perrin S. Anxiety disorders in children and adolescents with autistic spectrum disorders: a meta-analysis. Clin Child Fam Psychol Rev. 2011;14(3):302-317.

26. Steensel FJA, van Bogels SM, Wood JJ. Autism spectrum traits in children with anxiety disorders. J Autism Dev Disord. 2013;43(2): 361-370.

27. Ung D, Wood JJ, Ehrenreich-May J, et al. Clinical characteristics of high-functioning youth with autism spectrum disorders and anxiety. Neuropsychiatry (London). 2013;3(2):doi:10.2217/npy.13.9.

28. Renno P, Wood JJ. Discriminant and convergent validity of the anxiety construct in children with autism spectrum disorders. J Autism Dev Disord. 2013;43(9):2135-2146.
29. Sodian B, Frith U. Deception and sabotage in autistic, retarded and normal children. J Child Psychol Psychiatry. 1992;33(3):591-605.

30. Walsh P, Elsabbagh M, Bolton P, Singh I. In search of biomarkers for autism: scientific, social and ethical challenges. Nat Rev Neurosci. 2011;12(10):603-612.

31. Braida D, Guerini FR, Ponzoni L, et al. Association between SNAP-25 gene polymorphisms and cognition in autism: functional consequences and potential therapeutic strategies. Transl Psychiatry. 2015;5:e500.

32. Guerini FR, Bolognesi E, Chiappedi M, et al. HLA polymorphisms in Italian children with autism spectrum disorders: results of a family based linkage study. J Neuroimmunol. 2011;230(1-2):135-142.

33. Saresella M, Piancone F, Marventano I, et al. Multiple inflammasome complexes are activated in autistic spectrum disorders. Brain Behav Immun. 2016;57:125-133.
Neuropsychiatric Disease and Treatment

\section{Publish your work in this journal}

Neuropsychiatric Disease and Treatment is an international, peerreviewed journal of clinical therapeutics and pharmacology focusing on concise rapid reporting of clinical or pre-clinical studies on a range of neuropsychiatric and neurological disorders. This journal is indexed on PubMed Central, the 'PsycINFO' database and CAS,

\section{Dovepress}

and is the official journal of The International Neuropsychiatric Association (INA). The manuscript management system is completely online and includes a very quick and fair peer-review system, which is all easy to use. Visit http://www.dovepress.com/testimonials.php to read real quotes from published authors.

Submit your manuscript here: http://www.dovepress.com/neuropsychiatric-disease-and-treatment-journal 